\title{
A Novel Approach to Find Optimal Path by using Firefly Routing Algorithm in WSN
}

\author{
M.Nanthini, Rabin Kanisha K.R, V.Vakula, T.Vinothini, SrigithaS.Nath
}

\begin{abstract}
Wireless Sensor Networks are appropriate for many applications such as agriculture, smart phones, automation and disaster reduction. In general, medium access control protocol (MAC) plays a vital role in WSN by informing the network when and how to access a medium and as a result it reduces the energy consumption. In wireless sensor networks in the star topology consistent $802.15 .4 \mathrm{k}$ standard in which sensors could neglect to report detecting data to the get to point because of impermanent checks that disorder the connection with the access point. In this paper we discuss the connectivity and information loss of wireless environments. We categorize the work First; we study general connectivity requirements in relay networks. Second, to avoid information loss and to restore the proper connectivity. It can be performed by firefly algorithm with localizability aided localization protocol (F-LSL). It depends on the device of stochastic geometry and specifically, on Poisson direct procedures toward look for the tradeoff, which emerges from the determination of a subset of transfer hubs and the vital transmitted power that transfers need to use to reestablish arrange network.

Keywords: Delay, F-LSL (Firefly algorithm with Location Sustained localizability protocol), Network coverage area, Network lifetime, Packet loss, Throughput, Wireless Sensor Networks
\end{abstract}

\section{INTRODUCTION}

AwSN hoist in giving dependable correspondence however thinks that it's hard to beat the idleness which is the significant obstacle to accomplish the precision in multi casting. There are several types of routing protocols that consider the energy efficiency of sensors in their routing activities. Generally, sensor networks seem to be small in size,

Manuscript received on July 14, 2021.

Revised Manuscript received on July 30, 2021.

Manuscript published on July 30, 2021.

* Correspondence Author

M.Nanthini, Assistant Professor, Department of Electronics and Communication Engineering, Saveetha Engineering College, Chennai, India.vmnandhini0594@gmail.com

Rabin kanisha K.R, Assistant Professor, Department of Electronics and Communication Engineering, Saveetha Engineering College, Chennai, India. rabinkanisha95@gmail.com

V.Vakula*, Assistant Professor, Department of Electronics and Communication Engineering, Saveetha engineering college, Chennai, India. vakula2596@gmail.com

T.Vinothini, Assistant Professor, Department of Electronics and Communication Engineering, Saveetha Engineering College, Chennai, India. Vmnandhini0594@gmail.com

Dr.SrigithaS.Nath, HoD, Department of Electronics and Communication Engineering, Saveetha Engineering College, Chennai, India. sirgithapramodh2000@gmail.com

(C) The Authors. Published by Blue Eyes Intelligence Engineering and Sciences Publication (BEIESP). This is an open access article under the CC BY-NC-ND license (http://creativecommons.org/licenses/by-nc-nd/4.0/) capability of wireless, battery power saving ability, smart sensor nodes. Sensor nodes from the sensor networks have communication capabilities in many fields. They are manufactured by the concept of MEMS. For real time application time division multiple accesses are best suited. Because it prevents radio interference and reduces energy consumption. Generally routing protocols are classified based on location, data centric, hierarchical, mobility, multi-path, heterogeneity and QOS based protocols. In the case of hierarchical networks where the nodes are grouped into clusters and among them a particular node is elected as a cluster head. The cluster head plays a vital role in coordinating activities within the cluster and for forwarding information between clusters. Clustering technique helps to reduce energy Consumption and increase the network lifetime by achieving a high delivery ratio. Our F-LSL protocol is location based hierarchical routing protocol, used for reducing the delay and packet loss of a system with good throughput ratio.

The idleness is primarily because of information aggregation in the bunch head. The assault against the information conglomeration brings about faking the detected information or copying the first message with no data in it. As the information accumulating occurred with the fake or noxious code, the vitality utilization and transmission stack increments in remote sensor arrange environments. Hubs in the system transmit estimations to guardians subject to the obstruction limitations which is hub selective. Guardians meld data and transmit it to their folks. At that point the Sink registers the entirety which it got from its leaf hubs.

The WSN likewise upgrades the correspondence by finding the most limited separation between the sensor hubs. This can be accomplished through calculations accessible in the multi-cast directing convention.

\section{EXISTING SYSTEM}

The Zigbee (IEEE 802.15.4k) standard characterizes PHY and MAC layers diagnosis to support Low Energy Critical Infrastructure Monitoring networks (LECIM). Channel time is composed in super frames, with each isolated in a few sub-reference point intervals in addition to a discretionary latent period demarcated by carrying of signal casings transmitted by the AP. Signals do generally organize data, and also time synchronization for arranged gadgets. The transmission of a guide is trailed by a Contention Access Period and a Contention Free Period. Amid the CAP, transporters sense different access with crash evasion is utilized to transmit command outlines for affiliation and asset reservations inside the CFP.
Published By:

Blue Eyes Intelligence Engineering and Sciences Publication

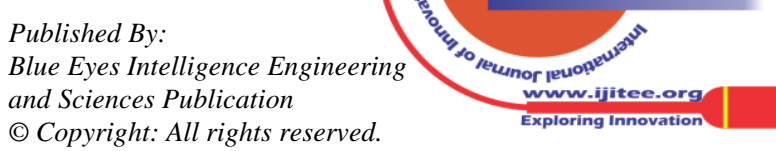


The CFP is TDMA based and is isolated into guaranteed time spaces. Amid one GTS, a stand out sensor is permitted to speak with the AP. Optimization issue is a standout amongst the most difficult issues in the field of operation research. The objective of the advancement issue is to locate the arrangement of factors that outcomes into the ideal estimation of the goal work, among every one of those qualities that fulfill the limitations.

\section{LITERATURE SURVEY}

A. Numerous new sorts of improvement calculations have been investigated. One of them is a nature-propelled sort. Calculations of this sort are, for example, a subterranean insect province improvement calculation proposed. This has been effectively connected to booking issues. ACO is motivated by the ants' social conduct of discovering their nourishment sources and the most limited ways to their settlement, set apart by their discharged pheromone. Another case of this sort of calculations is a molecule swarm streamlining calculation. PSO depends on the swarming conduct of schools of fish and winged animals in nature. PSO has been effectively connected to a wind vitality determining issue where wind vitality is evaluated in light of two meta-heuristic characteristics of swarm insight. A firefly calculation is yet another illustration. It is a populace-based calculation motivated Optimal path Using F-LSL

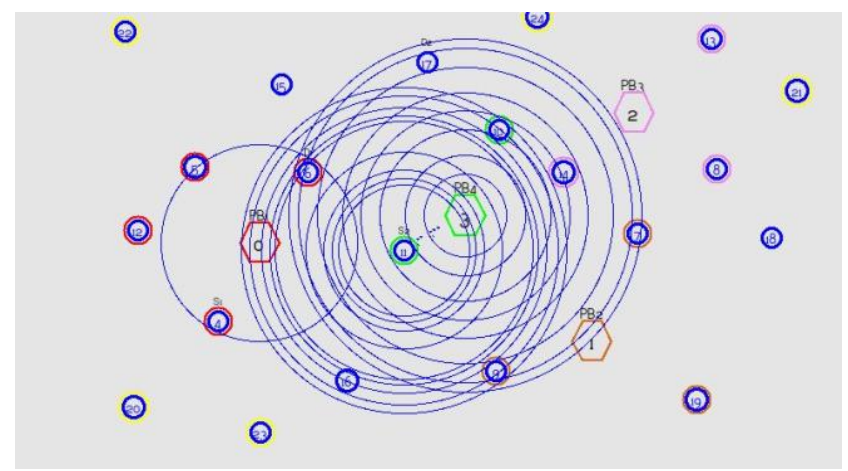

Fig.3. Location appraising

By the social conduct of fireflies. Fireflies impart by blazing their light. Dimmer fireflies are pulled in to brighter ones and move towards them to mate. Firefly algorithm is generally used to tackle unwavering quality and repetition issues.

\section{PROPOSED SYSTEM}

In our proposed work first the sensor networks form a clustered structure then determine their zone when the nodes in a network are either inter or intra zone nodes. This comprises three categories.

- Localization using LSL

- Localizability Testing then

- F-LSL

\section{A. Architecture}

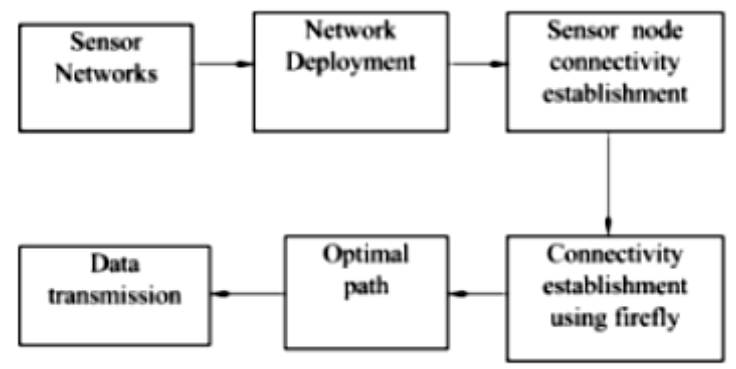

Fig.1. Proposed Architecture of F-LSL

B. Localization using LSL

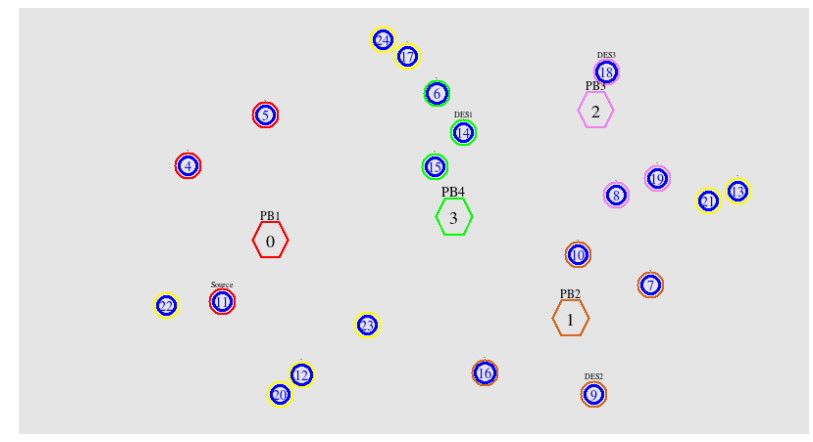

Fig: 2 Node Localization

The network access link from sensors and its access point is defected by some other obstacles. It can be recovered by locality based heterogeneous routing protocol namely F-LSL (Firefly algorithm with Location sustained localizability). This protocol can easily adjust the network nodes for this capability of location hierarchical. LSL is a premier stateless routing protocol to improve the performance of routing.

\section{Location appraising:}

It is a method to add and accommodate the revamping implementation. Generally, this testing represents the embryonic network deployment then these outcomes are used to mandate ensuing adjustment. Next adaptive positions are rationalized for topographic routing. All regular beacons proliferate effectively cross the link for widest networks for the purpose of testing the sensing region.

In this testing users can easily deploy the sensor nodes in a sensor region. It helps to reduce packet loss and find the optimal path in the sensor network. F-LSL protocol to localize all nodes and create awareness of each and every node. LSL can virtually manage to regulate the nodes in a network, while conventional approach could only make in determinate up-licks. When the application is deployed in the network, due to some structured or habitat factors are unpredictable. It may be not ready for location appraising. Hence, node location appraising method is led foremost in LSL, which identifies localizable and non-localizable nodes in a network for further adjustment and analyzes the distance graph into two different nodes. These nodes are separated and arranged in a tree structure and then commonly specified one root. LSL was notified in appraising the nodes and its positions in the integral tree. All the non-localizable nodes are arranged in one circle.

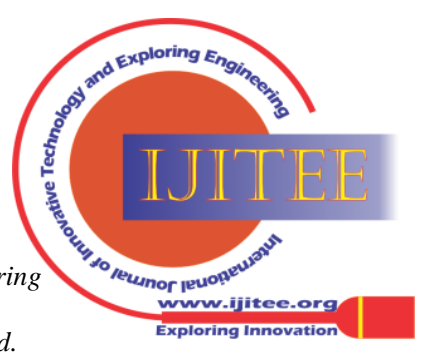




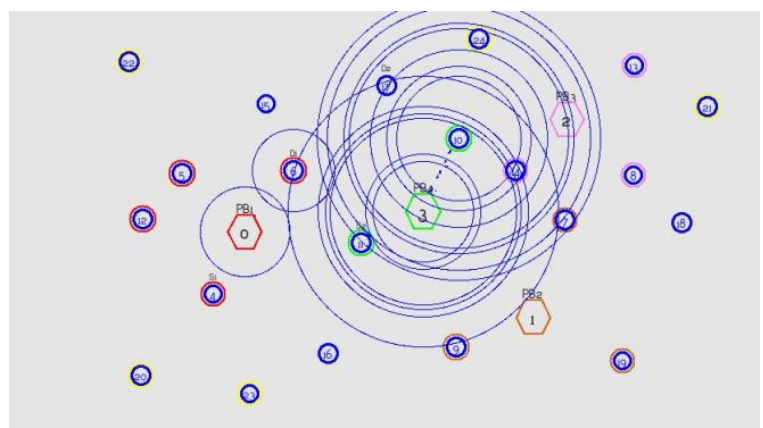

Fig.4.Find Optimal path using F-LSL

In the proposed approach each and every node finds the primary concept of wireless sensor nodes is to separate the control plane which is the decision-making component of the network, from the data plane which handles the actual data movement in the network. This separation of data plane from control plane making it possible to control or monitor a network from a centralized controller view. By using this above process, we can find the optimal path that means which node is very close to destination.

\section{SYSTEM ANALYSIS}

\section{A. Algorithm}

2.Deploy the sensor nodes in the network environment.

3. Generate initial population using firefly and LSL rules based on the light intensity.

4. Based on the distance, evaluate new solutions and update the intensity of the sensor.

5. Each and every node ranks it.

6. Finally choose the optimize path.

\section{B.F-LSL}

In traditional networks, routers and switches have individual decision-making capabilities provided by specific sensor nodes in the wireless sensor nodes running on them. Since each vendor follows different software specifications, functionalities of all these devices differ from each other and can't be changed uniformly across the network as different vendor components require different changes making the reconfiguration process very complex. In, data planes are cheap commodity forwarding devices without any decision-making capabilities. SDN allows having a centralized control plane where all the decisions are taken for the date plane.

This centralized control plane uses the Open Flow protocol to communicate with the data plane. Network applications are implemented on top of the control plane by using a combination of flow table entries. Controller takes the flow-based routing decisions and each controller decision are made as a new rule entry or overwrites an existing rule in the flow table. Ordinarily Members with high wellness will play out their employments proficiently and achieve excellent accomplishments. However, the low-quality individuals can't achieve such fantastic accomplishments. The nature of every sensor is assessed by individuals from the separate sensor.

The likelihood of acquiring qualified and reasonable arrangements in qualified populaces is high. In view of firefly calculation that to enhance the execution of the operators in
1. Generate network nodes.

deciding more proper arrangements by altering them (by rolling out improvement in the specialists' circumstances), builds up the sensor of firefly's general sensor, thus the likelihood of finding the ideal arrangement can be expanded. For this procedure, they have utilized which records and watch every one of the points of interest of the Fireflies conduct. These status specialists that must be changed in their circumstances by hopping into new circumstances. The procedure of basic leadership is executed on the inquiry operators to acknowledge whether any specialist needs to utilize the bounce alternative or not.

\section{RESULTS AND DISCUSSIONS}

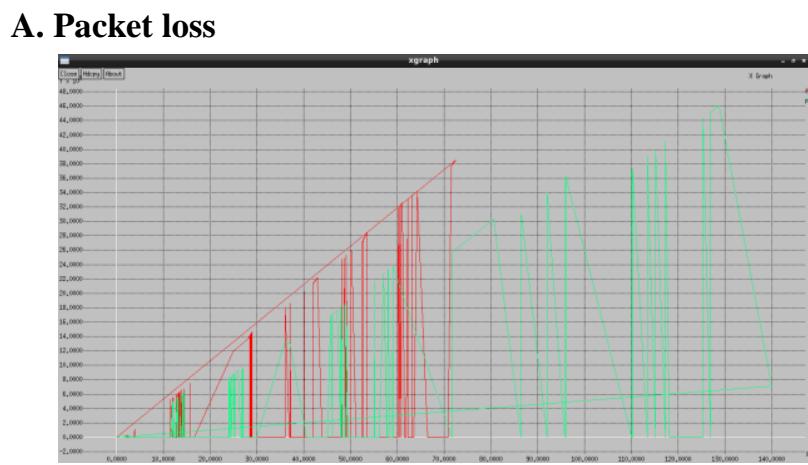

Fig.5. Packet loss

The graph analysis of existing and proposed analysis cleanly shows the loss of communication can be reduced with the firefly and LSL rules. In this communication information shared by one node to another it totally reduces the loss of communication in the sensor nodes.

\section{B. Delay}

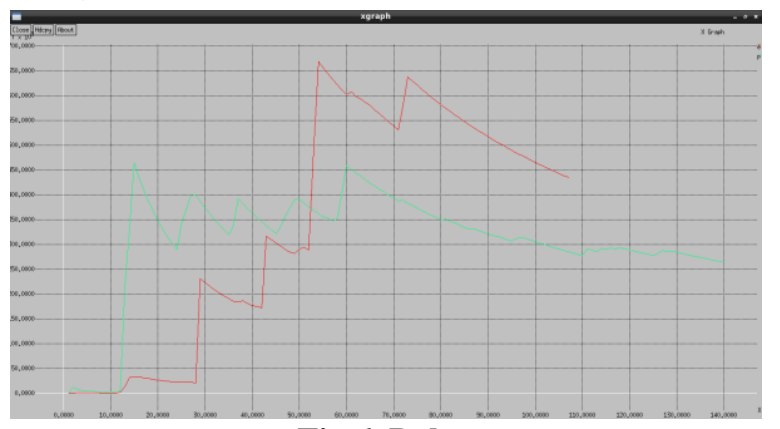

Fig.6. Delay

Analysis of delay using time is refinement by using the firefly algorithm. The computer Simulation outcomes secured in wireless sensor networks with multiple numbers of sensor nodes are adequate to demonstrate the scalability. As different time delays are related, sensor nodes in different algorithms can be monitored. These delays have to be taken away from verified measurement, to get the exact values of sensor nodes.

\section{Throughput}

In this graph the throughput can be increasing and automatically it reduces the delay in the wireless Sensor networks. Approximately it can be improved from 10 to $15 \%$.

Published By:

Blue Eyes Intelligence Engineering and Sciences Publication (C) Copyright: All rights reserved. 


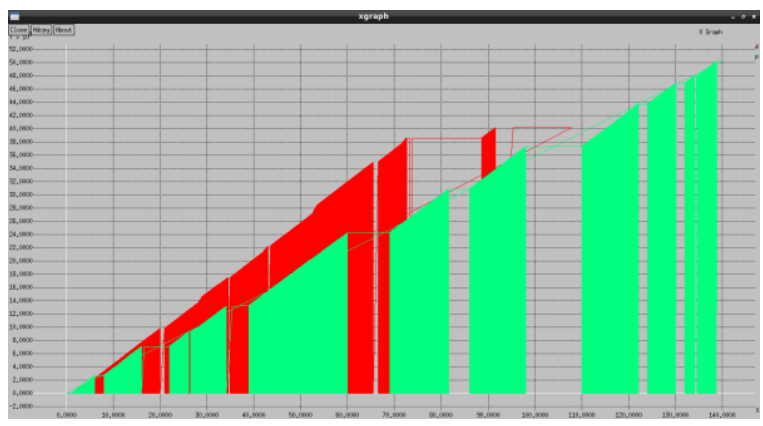

Fig.7. Throughput

D. Network coverage area

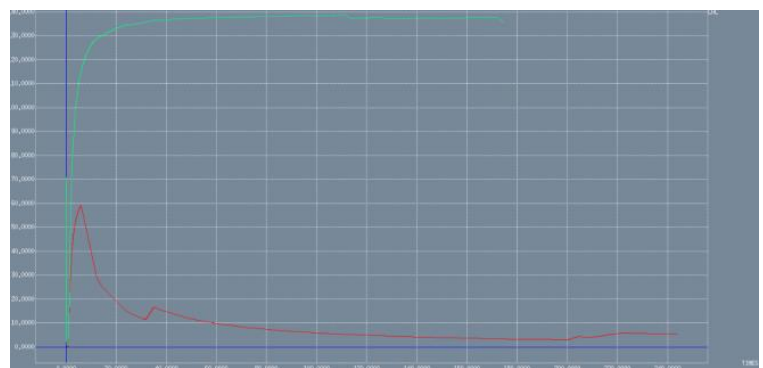

Fig.8. Network coverage area

Next the above graph represents network coverage. By using PSO we can increase the coverage area with low power consumption. It is the important key feature. It determines the capability of communication between sensor nodes in a network.

\section{E. Network lifetime}

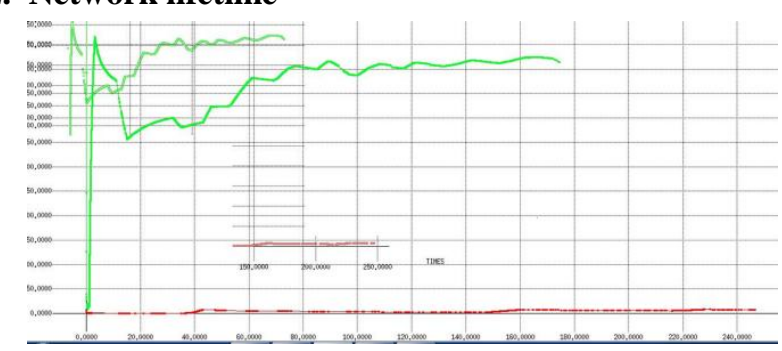

Fig.9.Network lifetime

By using F-LSL optimal paths can be easily achieved. It improves the network lifetime because LSL maintains the neighbor node position. If any node does not have a capability of transmitting data packets it is used as the next preferable node to communication for transferring the data packets. So the network does not have more number of collide packets. The life time will be improved.

\section{CONCLUSION}

We have proposed the LSL and firefly algorithm to optimize the path in the sensor nodes in the wireless environment. A novel convention to recuperate from availability misfortune when the immediate connection between at least one sensor and the access point is connected by the sudden appearance of big hindrances. We have examined the experimentation in the wireless environment to optimize the path and reduce the loss of information. Whereby our examination we figured out how to distinguish the arrangement of compromise focuses between the powers that sensors need to spend to transmit s-guide outlines and the topological change because of the way that the star topology transforms into neighborhood work systems. As future work we have wanted to develop the experimentation of this study, contrasting the execution of the proposed EDFA with the one displayed by a few as of late proposed metaheuristic, for example, to improve the throughput and delay to utilize the novel course in the wireless sensor networks.

\section{APPENDIX}

It is optional. Appendixes, if needed, appear before the acknowledgment.

\section{ACKNOWLEDGMENT}

It is optional. The preferred spelling of the word "acknowledgment" in American English is without an "e" after the "g." Use the singular heading even if you have many acknowledgments. Avoid expressions such as "One of us (S.B.A.) would like to thank ... ." Instead, write "F. A. Author thanks "Sponsor and financial support acknowledgments are placed in the unnumbered footnote on the first page.

\section{REFERENCES}

1. A.Banerjee, D.N.Ghosh and S. Das, "Repulsion-Propulsion Firefly Algorithm with Fast Convergence to Solve Highly Multimodal Problems," 2016 6th International Conference on IT Convergence and Security (ICITCS), Prague, Czech Republic, 2016, pp. 1-6.

2. K. Keskin and A. Karamancioğlu, "Application of Firefly algorithm to train operation," 2016 IEEE 8th International Conference on Intelligent Systems (IS), Sofia, Bulgaria, 2016, pp. 692-697.

3. NingXu, Sumit Rangwala, Ganesan, Alan Broad, Ramesh Krishna Kant Govindan, Chintalapudi, and Deborahwireless sensor network for structural monitoring. Proceedings of the2nd Deepak Estrin. InSenSys '04:international conference on Embedded networked sensor systems, New York, NY, USA.,pp 13-24. 2004.

4. BingnanPei; HaZhang; TengdaPei Hongyan Wang:Firefly algorithm optimization based WSN localization algorithm.2015 International Conference on Information and Communications Technologies (ICT 2015).

5. $\quad$ S.K.L. V. Sai Prakash;kondampalli S.Rami Reddy firefly inspired Kondapalli S. RamiReddy Firefly inspired energy aware cluster based tree formation in WSN. 2nd International Conference on Information and Communication Technology (IcoICT)2014.

6. Yi sun; Qing jiang; kai zhang.A clustering scheme for reachback firlefly synchroncity in wireless sensor networks. 2012 3rd IEEE International Conference on Network Infrastructure and Digital Content

7. Jalawi Sulaiman Alshudukhi;Zeyad Ghaleb Al-Mekhlafi;Mohammad T.Alshammari Badiea Abdulkarem Mohammend. Desynchronization Traveling Wave Pulse-Coupled-Oscillator Algorithm Using a Self-Organizing Scheme for Energy-Efficient Wireless Sensor $\underline{\text { Networks }}$

8. S. Gope, A. K. Goswami, P. K. Tiwari and S. Deb, "Generator rescheduling for congestion management using Firefly Algorithm," 2015 International Conference on Energy Systems and Applications, Pune, 2015, pp. 40-44.

9. K. K. Bhattacharjee and S. P. Sarmah, "A binary firefly algorithm for knapsack problems," Industrial Engineering and Engineering Management (IEEM), 2015 IEEE International Conference on, Singapore, 2015, pp. 73-77.

10. C. Hao, P. Song, J. Wu and C. Yang, "Multistage cycle synchronization strategy for WSN based on firefly-inspired algorithm," Information and Automation, 2015 IEEE International Conference on, Lijiang, 2015, pp. 1714-1717.

11. Shachi Battar; Rakesh Kumar A Hybrid Approach to Increase Network Lifetime in WSN Using PSO and Firefly Optimization. IEEE International Conference on Electrical, Computer and Communication Technologies (ICECCT)2019.

Published By:

Blue Eyes Intelligence Engineering and Sciences Publication (C) Copyright: All rights reserved. 
12. Navpreet Kaur Samra;Ramanpreet Kaur;Bikaram pal karur A Novel approach for energy efficient and congestion control in WBAN. 6th International Conference on Computing for Sustainable Global Development (india com) 2019.

13. D. Sánchez, P. Melin, J. Carpio and H. Puga, \&quot;A firefly algorithm for modular granular neural networks optimization applied to iris recognition,\&quot; 2016 International Joint Conference on Neural Networks (IJCNN),Vancouver, BC, Canada, 2016, pp. 139-144.

14. A Novel Sensor Deployment Approach Using Fruit Fly Optimization Algorithm in Wireless Sensor Networks. 2015 IEEE Trustcom/BigDataSE/ISPA.Huan Zhao, Qian Zhang, Liang Zhang, Yan Wang.

15. M. Nandhini, P. Priya. "A hybrid routing algorithm for secure environmental monitoring system in WSN", 2017 International Conference on Communication and Signal Processing (ICCSP), 2017

\section{AUTHORS PROFILE}

M.Nanthini, M.E, Assistant Professor from saveetha engineering college, chennai

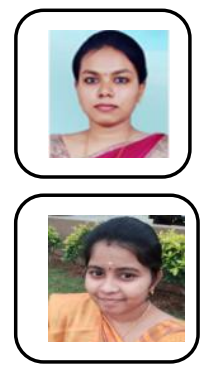

Rabin Kanisha R.K, M.E, Assistant Professor from saveetha engineering college, Chennai. Published by Design and analysis of broadband impedance matching network for Rectenna.

V.Vakula, M.E, Assistant Professor from saveetha engineering college, Chennai.

- $\quad$ Project Title :Open Source Tool for Land Use Monitoring System using Geo SpatialData Agency: DST-NRDMS Duration :2 Years (2018 -2020) Project Cost: Rs.5,60,000 Role PROJECT ASSISTANT Employer Bannari Amman Institute of Technology.

- $\quad$ 'Automatic 3D Building Footprint Extraction from High Resolution Satellite Image using OSM' in International Journal of Innovative Technology and Exploring Engineering (IJTEE), ISSN: 2278-3075 (Online), Volume-8 Issue-12, October 2019, PageNo.1642-1646.

- 'A Research on Identifying Building Object from Satellite Images' authored by "' which has published in 'International Journal of Engineering and Advanced Technology (IJEAT)', ISSN: 2249- 8958 ,Volume-8 Issue-6S3, September2019, PageNo.1091- 1094.

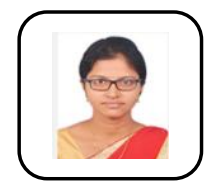

T.Vinothini, M.E, Assistant Professor from saveetha engineering college, chennai

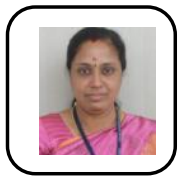

Dr.SirigthaS. Nath, HoD / Department of Electronics and communication System from saveetha engineering college, chennai

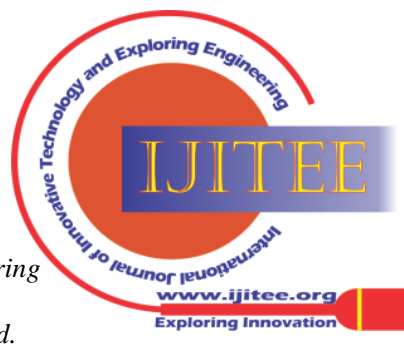

